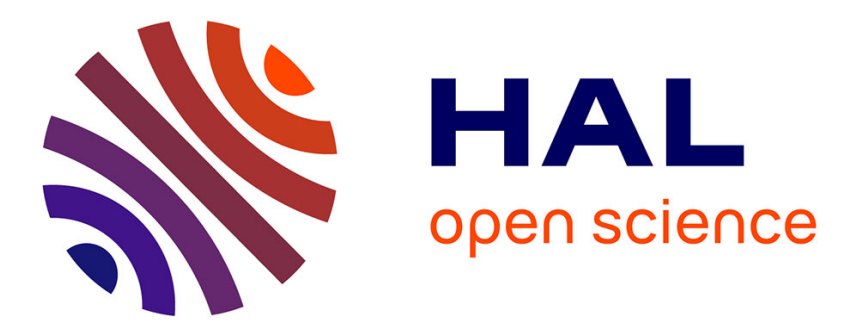

\title{
Juger la pertinence de la régulation des comportements déviants en Éducation Physique et Sportive: une comparaison entre enseignants titulaires et stagiaires
} Éric Fruchart, Patricia Rulence-Pâques

\section{- To cite this version:}

Éric Fruchart, Patricia Rulence-Pâques. Juger la pertinence de la régulation des comportements déviants en Éducation Physique et Sportive: une comparaison entre enseignants titulaires et stagiaires. Movement \& Sport Sciences - Science \& Motricité, 2018, pp.9 - 21. 10.1051/sm/2018002 . hal03425978

HAL Id: hal-03425978

https:/ /hal-univ-perp.archives-ouvertes.fr/hal-03425978

Submitted on 11 Nov 2021

HAL is a multi-disciplinary open access archive for the deposit and dissemination of scientific research documents, whether they are published or not. The documents may come from teaching and research institutions in France or abroad, or from public or private research centers.
L'archive ouverte pluridisciplinaire HAL, est destinée au dépôt et à la diffusion de documents scientifiques de niveau recherche, publiés ou non, émanant des établissements d'enseignement et de recherche français ou étrangers, des laboratoires publics ou privés. 


\title{
Juger la pertinence de la régulation des comportements déviants en Éducation Physique et Sportive : une comparaison entre enseignants titulaires et stagiaires
}

\author{
Éric Fruchart ${ }^{1, *}$ et Patricia Rulence-Pâques ${ }^{2}$ \\ ${ }^{1}$ Laboratoire Européen Performance Santé Altitude-EA 4604, Université de Perpignan Via Domitia, Département STAPS, \\ 66120 Font-Romeu, France \\ ${ }^{2}$ Laboratoire PSITEC-Psychologie: Temps, Interactions, Émotions, Cognition. Axe 2 : Éducation et Société-EA 4072, \\ Université de Lille III, Domaine du Pont de bois, BP 60149, 59653 Villeneuve d'Ascq cedex, France
}

Reçu le 12 octobre 2016, Accepté le 26 septembre 2017

\begin{abstract}
Résumé-L'objectif de cette étude était de mettre en évidence les différences entre 30 enseignants titulaires et 24 enseignants stagiaires pour juger la pertinence de la régulation de comportements déviants au cours d'une leçon en Éducation Physique et Sportive. Les participants ont indiqué leur jugement de pertinence dans 48 scénarios construits à partir du croisement de cinq informations contextuelles : la stratégie de régulation mise en place, le comportement déviant auquel l'enseignant est confronté, le niveau de la classe de l'élève, l'habitude de l'élève à adopter ce type de comportement, le comportement habituel de l'équipe d'Éducation Physique et Sportive de l'enseignant. Les résultats montrent que les stagiaires se sont distingués des titulaires dans la combinaison des informations contextuelles prises en compte dans leurs jugements.
\end{abstract}

Mots clés : enseignants, jugement, régulation, comportements déviants, Éducation Physique et Sportive

\begin{abstract}
Judging the relevance of deviant behaviours regulation in Physical Education: a comparison between trainee teachers and tenure teachers. The aim of our study was to highlight the differences between 30 tenure teachers and 24 trainee teachers to judge the relevance of deviant behaviours regulation during a lesson in Physical Education. The participants indicated their judgment of relevance in 48 scenarios constructed based on the combination of five contextual information cues: the control strategy implementation, the deviant behaviours which the teacher is confronted to, the level of the student's class, the student's habit to adopt this type of behaviour, the habitual behaviour of the teacher's team of Physical Education. The results showed that trainee teachers and tenure teachers used differently the contextual information cues in their judgments.
\end{abstract}

Key words: teachers, judgment, regulation, deviant behaviour, Physical Education

Dans le domaine de l'Éducation Physique et Sportive, la régulation des comportements déviants constitue une des préoccupations principales de l'activité des enseignants et des stagiaires en formation (Cruz, 2006). En effet, la confrontation à ce type de comportements peut générer du stress chez les enseignants et provoquer des conséquences néfastes pour leur santé (Lavay, Henderson, French, \& Gurthrie, 2012). Certains enseignants en Éducation Physique et Sportive n'hésitent pas à se réorienter professionnellement lorsqu'ils sont trop souvent confrontés à des conflits émanant de comportements non appropriés de la part des élèves (Petiot, Visioli \& Desbiens,

\footnotetext{
*Auteur correspondant : eric.fruchart@univ-perp.fr
}

2015). En fin de formation, les stagiaires en Éducation Physique et Sportive sont très rapidement au contact de cette réalité professionnelle et se trouvent parfois désemparés, en difficulté à gérer leur classe, ce qui a pour incidence une perte potentielle du contrôle de leur classe ou des pertes de temps (Desbiens et al., 2008). En outre, le contexte spécifique de l'enseignement de l'Éducation Physique et Sportive (e.g., environnement ouvert, nombre d'élèves dans les classes) peut compliquer considérablement la gestion des comportements déviants (Tsouloupas, Carson, \& MacGregor, 2014).

Cette question de régulation des comportements déviants constitue également un véritable enjeu éducatif et civique (Desbiens et al., 2011). Les comportements 
déviants perturbent les apprentissages scolaires des autres élèves (Morin \& Battalio, 2004) et gênent à la mise en place des situations d'apprentissage (Supaporn, Dodds, \& Griffin, 2003). L'un des objectifs de l'Éducation Physique et Sportive est le développement de la citoyenneté des individus et, il est indispensable que les enseignants régulent ces comportements afin que leurs élèves sachent ultérieurement s'inscrire dans la société (Lewis, Romi, Qui, \& Katz, 2005). Pour ces raisons, il est nécessaire de comprendre la pertinence des stratégies utilisées par les enseignants pour réguler les comportements déviants des élèves dans un cours d'Éducation Physique et Sportive.

En Éducation Physique et Sportive, la notion de comportement déviant est différemment étudiée, considérée ou définie. Quel que soit le courant scientifique, elle s'apparente «à la perception par les enseignants d'un comportement inadapté au contexte dans lequel se déroule l'action, c'est-à-dire la transgression d'une règle socialement construite » (Ayme, Ferrand, \& Cogérino, 2011). Le contexte auquel l'enseignant est confronté tient alors une place centrale pour étudier la régulation des comportements déviants. Pour autant, la notion de contexte et la gestion des comportements déviants sont envisagées différemment selon les paradigmes scientifiques : le paradigme behavioriste ou processus produit, le paradigme de l'énaction et le paradigme cognitiviste.

Dans le paradigme behavioriste, les chercheurs étudient les «comportements perturbateurs » ou «incidents disciplinaires » des élèves et les stratégies mises en place par les enseignants en réaction à ces comportements à partir d'observations, questionnaires ou entretiens (e.g., Turcotte et al., 2008). Goyette, Doré et Dion (2000) ont souligné que les enseignants stagiaires ont tendance à utiliser des réactions directes (cœrcitives). Leur stratégie de régulation varie en fonction de l'intensité du comportement déviant. Lors de comportement déviant de forte intensité, les stratégies directes et indirectes (conciliation) sont complémentaires. Dervaux, Carlier et Gérard (2004) ont signalé qu'ils utilisent principalement des pédagogies autoritaires afin de contrôler au mieux la classe, ensuite des pédagogies interactives et enfin des pédagogies permissives. En revanche, la littérature souligne deux limites à ce paradigme: la difficulté d'interprétation des expériences vécues par les élèves ou enseignants et la difficulté d'explication des interactions entre ces acteurs (e.g., Vors \& Gal-Petitfaux, 2014).

Pour répondre à cette limite, le paradigme de l'énaction s'intéresse à l'émergence d'un monde signifiant au cours de l'histoire des interactions continues entre l'acteur et son environnement physique, social et culturel, et à la signification que chacun attribue à ce qu'il est en train de faire (Varela, 1989). L'approche écologique considère les comportements déviants en investissant la dynamique et les interactions à l'intérieur de la classe (e.g., Hastie \& Siedentop, 2006). Ils sont incorporés dans un système complexe de relations entre le maintien de l'ordre, la promotion de l'apprentissage et la socialisation de l'élève (Barker \& Annerstedt, 2014). Le maintien de l'ordre est alors considéré comme un ensemble de règles, routines et attentes des élèves dans l'optique de favoriser l'apprentissage. Cette approche insiste sur le fait que les tâches sociales et le maintien de l'ordre constituent deux composantes nécessaires à l'apprentissage (pour une revue en Éducation Physique et Sportive, voir Hastie \& Siedentop, 2006).

Reposant également sur le paradigme de l'énaction, l'approche de l'action située se réfère aux situations sociales et aux interactions de l'individu avec l'environnement pour comprendre les comportements déviants en Éducation Physique et Sportive. Les travaux en action située portent sur la cognition située dans laquelle le comportement déviant est envisagé à partir de l'expérience vécue des enseignants, et de la dimension subjective et singulière de l'activité (e.g., Vors \& Gal-Petitfaux, 2014). Les comportements déviants sont analysés en lien avec les «activités clandestines» (e.g., Guérin, Testevuide, \& Roncin, 2005), «les conflits» (e.g., Flavier, Bertone, Méard, \& Durand, 2002) ou encore le « rapport à la règle » qui s'instaure dans l'interaction entre les élèves et l'enseignant (e.g., Méard, Bertone, \& Flavier, 2008). Cette cognition située considère le contexte comme subjectif. Il se réfère à la signification donnée par les enseignants et/ou élèves à leurs actions et à la singularité des situations. Les comportements déviants sont indéterminés et s'étudient in situ. Ce courant souligne que la gestion des comportements déviants en contexte est complexe, et notamment que l'enseignant en Éducation Physique et Sportive consacre un temps important à gérer des transactions entre lui et les élèves à propos des règles en classe (Flavier et al., 2002 ; Méard et al., 2008). En effet, l'enseignant est un guide, un médiateur. C'est l'action qui donne du sens à la situation et l'activité cognitive est spécifique au contexte. Les changements de comportements ne sont possibles qu'à l'aide d'efforts de négociation ou d'actions de régulation (Méard et al., 2008). Cependant, l'approche de l'action située présente certaines limites. Notamment, les travaux ne s'appuient que sur quelques études de cas ; ils ne prennent pas en considération l'activité cognitive à l'origine de la mémorisation ou de l'automatisation dans les phases d'apprentissages ; ou encore ils mettent de côté les représentations cognitives dans la médiatisation de la pensée au plan de la compréhension de la régulation de l'action (Desbiens, 2010).

Le paradigme cognitif répond à ces limitations. L'approche cognitiviste porte plus particulièrement son attention sur les processus cognitifs comme le traitement de l'information, les croyances ou les pensées (e.g., Fernandez-Balboa, 1991). Le comportement déviant est abordé de manière plus standardisée, souvent à partir d'analyse de comportements prédéterminés. À l'inverse du courant de la cognition située, le contexte est standardisé et se compose d'un ensemble d'éléments ou informations issus de l'environnement scolaire. Cette approche cognitiviste appréhende alors les «stratégies cognitives » mises en place par les enseignants lorsqu'ils sont confrontés à des comportements déviants dans ce contexte standardisé (e.g., Fernandez-Balboa, 1991). Les travaux de Kearney (1988) montrent, à partir des 
contextes présentés a priori, que la stratégie de régulation utilisée par les enseignants stagiaires ou expérimentés diffère. Desbiens et al. (2011) ont démontré que les enseignants stagiaires emploient un panel limité de stratégies de régulation face aux comportements perturbateurs. Ceux-ci mettent plus souvent en place des stratégies de régulation de type autoritaire quel que soit le type de comportement déviant et s'appuient très peu sur une pédagogie interactive.

Dans l'approche cognitiviste du constructivisme (e.g., Piaget, 1970), Kearney (1988) a présenté des scénarios à des enseignants stagiaires et expérimentés pour différencier les schèmes cognitifs dans le cadre de la gestion des comportements déviants. Les enseignants expérimentés utilisent un panel de techniques plus important que les stagiaires pour réguler les comportements des élèves et sont capables d'adapter leur mode de régulation selon le type de comportements déviants. Ils emploient davantage les techniques interactives (e.g., feedback de l'enseignant) lorsqu'ils sont face à des comportements déviants passifs (e.g., refus de participer) alors qu'ils mettent en place principalement des techniques autoritaires (e.g., punir l'élève) lorsqu'ils sont confrontés à des comportements actifs (e.g., frapper dans le matériel).

En appliquant la théorie socio-cognitive de Bandura (1997), Tsouloupas, Carson et Matthews (2014) proposent d'étudier les comportements déviants en Éducation Physique et Sportive en se centrant sur l'efficacité des enseignants. De la même manière que dans l'approche cognitiviste, le contexte est standardisé. Utilisant une méthode qualitative, Tsouloupas, Carson et Matthews (2014) se sont intéressés à la manière dont les enseignants jugent leur capacité et leurs connaissances à agir efficacement face aux comportements perturbateurs. Des travaux développant cette approche ont montré que les enseignants moins expérimentés avaient plus de difficultés pour gérer les comportements déviants des élèves (e.g., Day, Sammons, Stobart, Kingston, \& Gu, 2007). A partir d'une approche multi-méthodologique (entretiens, questionnaires et observations), Almog et Shechtman (2007) ont souligné le manque de connaissances des enseignants novices et l'emploi prononcé pour les régulations restrictives afin de faire face aux problèmes comportementaux chez les élèves. De la Torre Cruz et Arias (2007) ont quant à eux soumis des questionnaires à des enseignants stagiaires et des enseignants en activité pour examiner leur croyance en leur efficacité d'enseignement. Ils ont montré que les stagiaires ignorent les ressources nécessaires pour gérer efficacement ces situations. Les années d'enseignement qui différencient les enseignants titulaires et stagiaires semblent donc un facteur primordial pour investir la question de la régulation des comportements déviants (Tsouloupas, Carson, \& Matthews, 2014).

Dans le courant de la cognition sociale, de la même manière, le contexte est envisagé de façon standardisée. Tsouloupas, Carson et Matthews (2014) soulignent la nécessité de prendre en compte des facteurs de différentes natures (e.g., environnementaux et personnels), et donc d'appliquer des approches multifactorielles, pour comprendre la gestion des comportements déviants en Éducation Physique et Sportive. Un rôle primordial est donné aux processus cognitifs, et notamment au jugement, pour analyser ce phénomène scolaire. En effet, les processus cognitifs développés par les enseignants, quand ils sont confrontés aux comportements déviants des élèves, affectent les actions mises en place pour gérer ces situations (e.g., Fernandez-Balboa, 1991). Pour autant, la question des processus de jugement, c'est-à-dire la manière dont les individus intègrent cognitivement différents facteurs (Anderson, 1996), pour estimer les capacités, connaissances, ou encore la pertinence d'une régulation n'a jamais été approfondie. Ainsi, nous proposons d'appliquer la théorie fonctionnelle de la cognition (Anderson, 1996) afin d'étudier la manière dont des enseignants, titulaires ou stagiaires, intègrent mentalement différentes informations contextuelles standardisées pour juger la pertinence de la régulation d'un enseignant confronté à un comportement déviant en Éducation Physique et Sportive.

La théorie fonctionnelle de la cognition (Anderson, 1996, 2008) s'appuie sur le postulat que toute perception, pensée ou action est orientée vers un but et dépend de l'intégration de différentes informations. Elle a pour but de découvrir quelles opérations d'algèbre cognitive les individus opèrent pour prendre une décision ou effectuer un jugement dans diverses situations. Elle renseigne sur les processus par lesquels diverses informations sont intégrées en un tout : le jugement. Elle permet la mise en évidence de lois psychologiques cognitives du traitement et de l'intégration de plusieurs stimuli. Cette algèbre cognitive porte non pas sur les stimuli proprement dits mais sur leur contrepartie psychologique, ou valeurs subjectives que l'individu leur accorde. La manière dont les informations vont être combinées par les individus va produire différentes règles cognitives simples: règles additives, disjonctives et conjonctives.

D'après Anderson (1996), lorsqu'un individu intègre des informations pour rendre un jugement final, un champ de stimuli externes (S1, S2...) subit trois opérations successives qui sont dirigées par les buts du sujet: (1) une opération de valuation qui fait correspondre aux stimuli donnés $\left(\mathrm{S}_{\mathrm{i}}\right)$, une représentation psychologique $\left(\mathrm{s}_{\mathrm{i}}\right) ;(2)$ une opération d'intégration qui agrège ces représentations psychologiques en une réponse implicite (r) ; et (3) une opération réponse qui rend observable la réponse implicite. Bien souvent il s'agit de la sélection d'une graduation sur une échelle de jugement.

La théorie fonctionnelle de la cognition a été utilisée pour étudier la manière dont les individus combinent différentes informations pour prendre une décision ou effectuer un jugement dans les domaines des activités physiques et sportives (e.g., Fruchart, Pâques, Dru, \& Mullet, 2010), de l'éducation (e.g., Rulence-Pâques \& Mullet, 1998) ou encore de la régulation des comportements déviants en sport (e.g., Fruchart \& RulencePâques, 2014). L'utilisation de la théorie fonctionnelle de la cognition (Anderson, 2008) présente un véritable intérêt. Lorsque l'on interroge les enseignants sur les 
facteurs susceptibles d'inférer leur jugement quant à la pertinence d'une régulation des comportements déviants dans le cadre scolaire, ils sont capables d'isoler les facteurs et d'exprimer s'ils sont individuellement importants pour eux. En revanche, si on leur demande dans un contexte standardisé présenté par plusieurs facteurs, de nous renseigner sur le poids qu'ils leur ont alloué ou sur la manière dont ils vont combiner mentalement ces facteurs pour rendre leur jugement de pertinence, ils seront dans l'incapacité de nous informer car la tâche cognitive est extrêmement complexe.

Nous avons considéré quatre facteurs pour caractériser le contexte standardisé de transition ou de période d'organisation d'une leçon d'Éducation Physique et Sportive dans laquelle les comportements déviants sont susceptibles d'apparaître. Le choix de ces facteurs a été guidé par la littérature sur la gestion des comportements déviants dans le domaine scolaire.

Le premier facteur est le mode de régulation des comportements déviants. À partir d'une enquête par entretiens individuels, Grimault-Leprince (2011) a questionné la façon dont les enseignants géraient les comportements déviants et a proposé une classification $a$ posteriori. Des enseignants utilisent principalement la sanction avec une sensibilité prononcée pour les normes et les règles; l'auteur les appelle les «enseignants formalistes». D'autres s'appuient sur la négociation (《les enseignants pragmatiques utilitaristes ») avec un souci d'efficacité pédagogique. Enfin, certains développent une attitude plus compréhensive centrée sur les difficultés liées à l'adolescence ( «les enseignants pragmatiques compréhensifs »). Sanction, négociation et compréhension sont des modes (ou stratégies) de régulation souvent liées aux exigences pédagogiques. Les enseignants les plus «formalistes » tentent, avec un succès inégal, d'imposer des règles rigides et recourent fréquemment à la sanction. Les plus «pragmatiques» trouvent des arrangements avec les élèves.

La nature du problème rencontré constitue le deuxième facteur. Les enseignants en Éducation Physique et Sportive sont confrontés au quotidien à beaucoup de comportements déviants allant de l'inattention au bavardage, de la tricherie à l'insolence jusqu'au comportement dangereux (e.g., Kulinna, Cothran, \& Regualos, 2006). Certains élèves peuvent décider de ne pas participer aux tâches prescrites par l'enseignant (Kulinna, Cothran, \& Regualos, 2003) ou encore de s'en prendre au matériel (Desbiens et al., 2011).

Le niveau de la classe est le troisième facteur. À partir d'entretiens qualitatifs, Grimault-Leprince (2011) informe qu'il serait plus difficile de gérer l'ordre dans des classes d'élèves plus jeunes que dans des classes d'élèves les plus âgés. Les classes composées de jeunes élèves sont décrites comme très remuantes, mais aussi très sensibles à l'autorité; Confrontés à des élèves plus âgés, la grande majorité des enseignants déclare rencontrer moins de difficultés. Ceci s'expliquerait par une responsabilisation des élèves les plus âgés avec une prise en considération des enjeux scolaires.
Le niveau d'habitude de l'élève au comportement déviant représente le quatrième facteur. Les enseignants sanctionneraient plus facilement des élèves qui sont souvent pénibles plutôt que des élèves qui le sont rarement (Grimault-Leprince, 2011). Étiquetés déviants par l'institution, les élèves difficiles peuvent mettre en place des stratégies de «résistance», avec des incidences sur la multiplication des conflits avec les enseignants. Les sanctions mettent alors en évidence les exigences pédagogiques et informent sur le climat de classe.

Le comportement habituel des collègues en Éducation Physique et Sportive est le cinquième facteur. À partir d'entretiens, Jelen (2014) souligne que, dans le cadre du projet pédagogique en Éducation Physique et Sportive, les enseignants sont amenés à échanger des connaissances dans la gestion et la prévention des comportements déviants. Le soutien des collègues est notamment une variable à interroger dans la perception des modes de régulation des comportements en classe (Petiot et al., 2015).

Aucune recherche en Éducation Physique et Sportive ne s'est intéressée à l'influence de tels facteurs sur les processus de jugement de pertinence de la régulation des comportements déviants. C'est pourquoi, l'objectif de cette étude a été de découvrir des différences dans la manière dont des enseignants stagiaires et des enseignants titulaires combinent différentes informations contextuelles pour juger la pertinence de la régulation des comportements déviants au cours d'une leçon en Éducation Physique et Sportive.

En nous appuyant sur les travaux précédemment présentés, nous avons émis deux hypothèses: (1) les participants prennent en compte différents facteurs liés au contexte de l'enseignement de l'Éducation Physique et Sportive pour juger la pertinence de la régulation: le type de stratégie de régulation, la nature du problème auquel l'enseignant est confronté, la classe de l'élève, l'habitude de l'élève à adopter ce type de comportement déviant, et l'emploi de cette stratégie de régulation par l'équipe d'Éducation Physique et Sportive à laquelle l'enseignant appartient; (2) les enseignants et les stagiaires ne prennent pas en compte les informations de la même manière pour estimer la pertinence de la régulation.

\section{Méthode}

\section{Sujets}

Cinquante-quatre personnes vivant dans le Nord de la France ont participé à cette étude. Trente étaient enseignants titulaires depuis au moins quatre ans dans les établissements du second degré, publics, non classés en zone prioritaire, rattachés à l'académie de Lille $\left(M_{\text {age }}=\right.$ 26,$33 ; S D=3,97$ ) et 24 étaient étudiants stagiaires dans l'académie lilloise inscrits en master 2 «Spécialité métiers de l'enseignement et de la formation en éducation physique et sportive, éduquer les Activités Physiques Sportives et Artistiques» de la faculté du Sport et de l'Éducation Physique et Sportive de l'Université d'Artois 
et de la faculté des Sciences du Sport et de l'Éducation Physique de l'Université de Lille $2\left(M_{\text {age }}=22,55 ; S D=\right.$ $1,24)$. Les participants ont été contactés par l'intermédiaire de leur établissement puis se sont inscrits sur la base du volontariat et du bénévolat pour apporter leur contribution à cette recherche.

\section{Matériel}

Le matériel a été constitué d'un questionnaire (Anderson, 1996) composé de 48 feuilles. Sur chaque feuille était présenté un scénario décrivant une situation rencontrée lors d'une leçon de handball dans le cadre d'un enseignement en Éducation Physique et Sportive suivi d'une question et d'une échelle de réponse. Chaque scénario contenait cinq informations. Le premier facteur concerne le mode de régulation et comporte les trois degrés correspondant à la classification de Grimault-Leprince (2011) qui sont la sanction, la négociation et la compréhension. Les autres facteurs sont la nature du problème rencontré (la perturbation ou le refus d'engagement dans l'activité), le niveau de la classe de l'élève (classe de sixième ou classe de terminale), l'habitude de l'élève à ce type de comportement (l'élève est un habitué de ce type de comportement ou c'est la première fois qu'il a ce type de comportement), le comportement habituel de l'équipe d'Éducation Physique et Sportive (elle emploie très rarement cette régulation ou elle emploie principalement cette régulation). Chacun de ces facteurs comporte deux modalités et correspond à un choix délibéré pour ne pas accroître le nombre total de scénarios. Le croisement de ces cinq facteurs a donné le plan factoriel suivant: $3 \times 2 \times 2 \times 2 \times 2=48$, soient 48 scénarios. Au sein de chaque scénario, le contexte a été figé en décrivant un regroupement en fin de séance d'Éducation Physique et Sportive afin que l'enseignant donne des consignes. La suite va correspondre au croisement des cinq variables choisies et sera différente dans chaque histoire. Chaque scénario a pris la forme suivante: «À la fin d'une leçon de handball avec une classe de sixième, Pierre regroupe ses élèves afin de leur transmettre les consignes d'un match à thème. La leçon s'est déroulée correctement, Pierre prend la parole dans le calme. Une fois les instructions transmises, Pierre donne la balle à un élève mais celui-ci frappe le ballon au pied pour montrer son mécontentement vis-à-vis de la constitution des équipes. Cet élève est un habitué de ce type de comportement. Pierre décide de réguler le comportement de l'élève en lui ordonnant de manière autoritaire de quitter son cours et en lui expliquant qu'il sera sanctionné de quatre heures de retenue. Les autres membres de l'équipe d'Éducation Physique et Sportive emploient très rarement la sanction pour réguler ce type de comportement. »

La question suivante était posée: Dans quelle mesure jugez-vous la régulation de Pierre pertinente? sous laquelle figurait une échelle de réponse de 11 graduations allant de Pas du tout pertinente à l'extrême gauche de l'échelle à Tout à fait pertinente à l'extrême droite.

\section{Déroulement}

Chaque participant a rempli le questionnaire de manière individuelle à l'université ou sur le lieu de travail. Selon la méthodologie de la théorie fonctionnelle de la cognition (Anderson, 1996), l'expérimentation s'est déroulée en deux phases. La première phase est appelée phase de familiarisation. Elle a pour objectif d'habituer les participants à la tâche et de stabiliser leurs jugements des scénarios constitués des différentes informations combinées. Au cours de cette phase, l'expérimentateur a expliqué en quoi consistait leur tâche et leur a soumis huit scénarios du plan factoriel, choisis de manière à ce que l'ensemble des différents facteurs et leurs modalités leur soient présentés. La tâche du participant a été de lire à voix haute chaque scénario puis de répondre à la question en cochant une graduation entre le Pas du tout pertinente et le Tout à fait pertinente en utilisant la totalité de l'échelle pour éviter les effets de plancher ou de plafond. Au cours de cette phase, toute question a pu être posée et les retours en arrière pour comparer les réponses ont été autorisés.

Durant la deuxième phase, appelée phase d'expérimentation, les sujets ont été confrontés à l'ensemble des 48 scénarios placés dans un ordre aléatoire. Leur tâche a été identique à celle de la phase de familiarisation mais les questions et les comparaisons de réponse n'étaient plus possibles.

\section{Considérations éthiques}

Les sujets étaient volontaires. Chacun d'entre eux a été averti que les conditions d'anonymat seraient garanties. Il leur a été précisé qu'aucun résultat ne serait transmis individuellement mais que les résultats principaux et l'analyse de toutes les réponses seraient source de publication à laquelle ils auraient accès. Les différents responsables des structures (établissements du second degré et facultés) dans lesquelles évoluaient les participants ont autorisé les passations des questionnaires.

\section{Méthodes d'analyse des résultats}

Seules les réponses des participants recueillies lors de la phase expérimentale ont été prises en compte, converties en valeurs numériques en comptant le nombre de graduations entre l'extrémité gauche de l'échelle de réponse et la graduation cochée par le sujet. Ces valeurs ont ensuite été soumises à des analyses statistiques (ANOVAs).

\section{Résultats}

Une ANOVA suivant le plan factoriel Population $x$ Stratégie $\times$ Problème $\times$ Classe $\times$ Habitude $\times$ Équipe d'Éducation Physique et Sportive a été menée sur l'ensemble des données. Des effets simples significatifs sont apparus pour quatre des cinq variables intra-sujets: la Stratégie, $F$ $(2,104)=644,67, \quad p<.000, \quad \eta_{\mathrm{p}}^{2}=.92 ; \quad$ la Classe, $\quad F$ $(1,52)=18,42, \quad p<.002, \quad \eta^{2}{ }_{\mathrm{p}}=.26 ; \quad$ l'Habitude, 
$F(1,52)=28,01, p<.000, \eta^{2}=.35 ;$ l'Équipe d'Éducation Physique et Sportive, $F(1,52)=278,91, p<.000, \eta^{2}=.84$. Seule la variable intra-sujets Problème n'a pas eu d'effet significatif, $F(1,52)=.10, p=.750, \eta_{\mathrm{p}}^{2}=.00$. La variable inter-sujets Population a eu un effet simple significatif, $F(1,52)=1595,55, p<.000, \eta_{\mathrm{p}}^{2}=.96$.

En ce qui concerne la variable Stratégie, les tests post hoc de Tukey ont révélé des différences significatives entre la sanction et la négociation $(p<.001)$ et entre la sanction et la compréhension $(p<.001)$. En revanche, il n'y avait pas de différence significative entre la négociation et la compréhension $(p=.21)$. L'interaction Population $\times$ Stratégie, $F(2,104)=56,95, p<.000, \eta^{2}{ }_{\mathrm{p}}=.53$, et l'interaction Population $\times$ Problème, $F(1,52)=12,17, \quad p<.001$, $\eta_{\mathrm{p}}^{2}=.19$, se sont avérées significatives. L'interaction Population $\times$ Classe, $F(1,52)=1,63, \quad p=.207, \quad \eta^{2}=.03$, l'interaction Population $\times$ Habitude, $\quad F(1,52)=0,01$, $p=.978, \eta_{\mathrm{p}}^{2}=.00$ et l'interaction Population $\times$ Équipe, $F(2,104)=3,08, p=.085, \eta^{2}{ }_{\mathrm{p}}=.06$, n'étaient pas significatives. L'interaction Population $\times$ Stratégie $\times$ Problème était significative, $F(2,104)=83,19, p<.000, \eta^{2}{ }_{\mathrm{p}}=.62$.

Une ANOVA suivant le plan factoriel Stratégie $\times$ Problème $\times$ Classe $\times$ Habitude $\times$ Équipe d'Éducation Physique et Sportive a été conduite sur les données recueillies auprès des deux populations, respectivement. Le tableau 1 présente les principaux résultats de l'ANOVA conduite sur les données des stagiaires et le tableau 2 présente les principaux résultats de l'ANOVA menée sur les données des titulaires. Dans l'ensemble, les jugements de pertinence des stagiaires $(M=3,29 ; S D=0,15)$ sont assez proches de ceux des titulaires $(M=3,11 ; S D=0,11)$. Le tableau 3 présente les moyennes et écart-types dans les deux échantillons.

La figure 1 présente l'interaction des facteurs Problème $\times$ Stratégie - celle-ci étant significative chez les titulaires et les stagiaires. Les résultats des stagiaires sont présentés sur la figure 1a. Les stagiaires n'ont pas jugé différemment la pertinence de la régulation selon le problème rencontré. Quel que soit le problème, les stagiaires différencient leur jugement quant au mode de régulation à adopter. Ils jugent plus pertinente une régulation par la sanction $(M=7,17 ; S D=0,22)$, puis par la négociation $(M=1,83 ; S D=0,16)$ et enfin par la compréhension $(M=0,87 ; S D=0,20)$. Les tests post hoc de Tukey se sont avérés significatifs entre la sanction et la négociation $(p<.001)$, entre la sanction et la compréhension $(p<.001)$, et entre la négociation et la compréhension $(p<.001)$.

Les résultats des titulaires sont présentés sur la figure $1 \mathrm{~b}$. La régulation par la sanction a été jugée plus pertinente pour un problème de perturbation $(M=7,80 ; S D=0,15)$ que pour un problème de refus $(M=2,26 ; S D=0,23)$. À l'inverse, la régulation par la négociation et par la compréhension a été jugée plus pertinente pour un problème de refus d'engagement dans l'activité $(M=2,51 ; S D=0,22$; $M=3,38 ; S D=0,26)$ que pour un problème de perturbation $(M=1,17 ; S D=0,12 ; M=0,94 ; S D=0,11)$. Les tests post hoc de Tukey ont confirmé la significativité de ces différences de jugement $\left(p_{\mathrm{s}}<.001\right)$.
Tableau 1. Principaux résultats de l'ANOVA menée sur les données des stagiaires.

\begin{tabular}{|c|c|c|c|c|c|c|c|}
\hline \multirow[b]{2}{*}{ Facteurs } & \multicolumn{2}{|c|}{ Effet } & \multicolumn{2}{|c|}{ Erreur } & \multirow[b]{2}{*}{$F$} & \multirow[b]{2}{*}{$p$} & \multirow[b]{2}{*}{$\eta_{\mathrm{p}}^{2}$} \\
\hline & $d f$ & $M S$ & $d f$ & $M S$ & & & \\
\hline Stratégie (S) & 2 & 4420,42 & 46 & 10,59 & 417,36 & .000 & .95 \\
\hline Problème $(\mathrm{P})$ & 1 & 29,71 & 23 & 7,98 & 3,72 & .066 & .14 \\
\hline Classe (C) & 1 & 15,82 & 23 & 5,14 & 3,08 & .093 & .12 \\
\hline Habitude (H) & 1 & 37,92 & 23 & 5,45 & 6,95 & .015 & .23 \\
\hline Équipe (É) & 1 & 150,95 & 23 & 1,06 & 142,48 & .000 & .86 \\
\hline $\mathrm{S} \times \mathrm{P}$ & 2 & 205,59 & 46 & 3,87 & 53,14 & .000 & .70 \\
\hline $\mathrm{S} \times \mathrm{C}$ & 2 & 259,82 & 46 & 4,23 & 61,46 & .000 & .73 \\
\hline $\mathrm{S} \times \mathrm{H}$ & 2 & 207,09 & 46 & 3,49 & 59,28 & .000 & .72 \\
\hline $\mathrm{S} \times \dot{\mathrm{E}}$ & 2 & 13,88 & 46 & 1,31 & 10,58 & .000 & .31 \\
\hline $\mathrm{P} \times \mathrm{C}$ & 1 & 2,62 & 23 & 4,09 & 0,64 & .431 & .03 \\
\hline $\mathrm{P} \times \mathrm{H}$ & 1 & 18,76 & 23 & 2,86 & 6,55 & .018 & .22 \\
\hline $\mathrm{P} \times \dot{\mathrm{E}}$ & 1 & 4,88 & 23 & 0,52 & 9,41 & .005 & .29 \\
\hline $\mathrm{C} \times \mathrm{H}$ & 1 & 87,23 & 23 & 3,87 & 22,54 & .000 & .49 \\
\hline $\mathrm{C} \times \mathrm{E}^{-}$ & 1 & 0,54 & 23 & 0,37 & 1,46 & .238 & .06 \\
\hline $\mathrm{H} \times \dot{\mathrm{E}}$ & 1 & 3,44 & 23 & 0,51 & 6,68 & .017 & .22 \\
\hline $\mathrm{S} \times \mathrm{P} \times \mathrm{C}$ & 2 & 129,26 & 46 & 3,19 & 40,53 & .000 & .64 \\
\hline $\mathrm{S} \times \mathrm{P} \times \mathrm{H}$ & 2 & 27,87 & 46 & 2,70 & 10,34 & .000 & .31 \\
\hline $\mathrm{S} \times \mathrm{P} \times \dot{\mathrm{E}}$ & 2 & 3,03 & 46 & 0,53 & 5,73 & .006 & .20 \\
\hline $\mathrm{S} \times \mathrm{C} \times \mathrm{H}$ & 2 & 54,86 & 46 & 3,81 & 14,40 & .000 & .39 \\
\hline $\mathrm{S} \times \mathrm{C} \times \dot{\mathrm{E}}$ & 2 & 1,00 & 46 & 0,41 & 2,45 & .098 & .10 \\
\hline $\mathrm{S} \times \mathrm{H} \times \mathrm{E}$ & 2 & 0,37 & 46 & 0,34 & 1,09 & .346 & .45 \\
\hline $\mathrm{P} \times \mathrm{C} \times \mathrm{H}$ & 1 & 1,92 & 23 & 2,30 & 0,83 & .371 & .03 \\
\hline $\mathrm{P} \times \mathrm{C} \times \mathrm{E}$ & 1 & 0,01 & 23 & 0,72 & 0,01 & .918 & .00 \\
\hline $\mathrm{P} \times \mathrm{H} \times \overline{\mathrm{E}}$ & 1 & 0,46 & 23 & 0,61 & 0,75 & .394 & .75 \\
\hline $\mathrm{C} \times \mathrm{H} \times \mathrm{E}$ & 1 & 0,63 & 23 & 0,50 & 1,25 & .274 & .05 \\
\hline
\end{tabular}

La majeure distinction entre les deux populations réside donc dans les jugements de régulation par la sanction. Pour les stagiaires, les jugements assez stables, allant de 6,52 à 7,82 sur l'échelle de jugement. Pour les titulaires, les jugements sont très variables, allant de 2,26 à 7,80 . Pour cette raison, une ANOVA suivant le plan factoriel Population $\times$ Problème $\times$ Classe $\times$ Habitude $\times$ Équipe d'Éducation Physique et Sportive a été menée sur les données provenant de la régulation par la sanction.

Des effets simples significatifs sont apparus pour les quatre variables intra-sujets: le Problème, $F(1,49)=$ $360,15, \quad p<.000, \eta^{2}{ }_{\mathrm{p}}=.88$; la Classe, $F(1,49)=71,30$, $p<.000, \eta_{\mathrm{p}}^{2}=.59 ;$ l'Habitude, $F(1,49)=295,62, p<.000$, $\eta^{2}=.86 ;$ l'Équipe d'Éducation Physique et Sportive, $F$ $(1,49)=91,15, p<.000, \eta^{2}{ }_{\mathrm{p}}=.65$. La variable inter-sujets Population a eu un effet simple significatif, $F$ $(1,49)=1989,60, p<.000, \eta^{2}=.98$. L'interaction Population $\times$ Problème, $F(1,49)=124,01, p<.000, \eta_{\mathrm{p}}^{2}=.72$, l'interaction Population $\times$ Classe, $F(1,49)=4,42, \quad p<.040$, $\eta^{2}{ }_{\mathrm{p}}=.08$ et l'interaction Population $\times$ Habitude, $F$ $(1,49)=8,56, p<.005, \eta^{2}{ }_{\mathrm{p}}=.19$ se sont avérées significatives. L'interaction Population $\times$ Équipe, $F(1,49)=0,48$, $p=.503, \eta_{\mathrm{p}}^{2}=.06$ n'était pas significative. L'interaction Population $\times$ Problème $\times$ Classe $\times$ Habitude était significative, $F(1,49)=31,33, p<.000, \eta^{2}{ }_{\mathrm{p}}=.39$. 
Tableau 2. Principaux résultats de l'ANOVA menée sur les données des titulaires.

\begin{tabular}{|c|c|c|c|c|c|c|c|}
\hline \multirow[b]{2}{*}{ Facteurs } & \multicolumn{2}{|r|}{ Effet } & \multicolumn{2}{|c|}{ Erreur } & \multirow[b]{2}{*}{$F$} & \multirow[b]{2}{*}{$p$} & \multirow[b]{2}{*}{$\eta^{2} \mathrm{p}$} \\
\hline & $d f$ & $M S$ & $d f$ & $M S$ & & & \\
\hline Stratégie (S) & 2 & 1786,96 & 58 & 8,21 & 217,61 & .000 & .88 \\
\hline Problème $(\mathrm{P})$ & 1 & 53,67 & 29 & 5,46 & 9,83 & .004 & .25 \\
\hline Classe (C) & 1 & 67,60 & 29 & 2,87 & 23,58 & .000 & .45 \\
\hline Habitude $(\mathrm{H})$ & 1 & 48,40 & 29 & 1,18 & 42,95 & .000 & .60 \\
\hline Équipe (E) & 1 & 123,67 & 29 & 0,92 & 133,67 & .000 & .82 \\
\hline $\mathrm{S} \times \mathrm{P}$ & 2 & 1900,94 & 58 & 5,32 & 357,39 & .000 & .93 \\
\hline $\mathrm{S} \times \mathrm{C}$ & 2 & 189,06 & 58 & 3,65 & 51,85 & .000 & .64 \\
\hline $\mathrm{S} \times \mathrm{H}$ & 2 & 401,64 & 58 & 1,94 & 207,51 & .000 & .88 \\
\hline $\mathrm{S} \times \dot{\mathrm{E}}$ & 2 & 5,85 & 58 & 1,07 & 5,44 & .007 & .16 \\
\hline $\mathrm{P} \times \mathrm{C}$ & 1 & 73,80 & 29 & 2,38 & 31,00 & .000 & .52 \\
\hline $\mathrm{P} \times \mathrm{H}$ & 1 & 126,02 & 29 & 1,92 & 65,49 & .000 & .70 \\
\hline $\mathrm{P} \times \overline{\mathrm{E}}$ & 1 & 10,00 & 29 & 0,49 & 20,59 & .000 & .42 \\
\hline $\mathrm{C} \times \mathrm{H}$ & 1 & 224,04 & 29 & 1,84 & 121,73 & .000 & .81 \\
\hline $\mathrm{C} \times \overline{\mathrm{E}}$ & 1 & 0,47 & 29 & 0,32 & 1,45 & .238 & .05 \\
\hline $\mathrm{H} \times \dot{\mathrm{E}}$ & 1 & 2,02 & 29 & 0,42 & 4,84 & .036 & .14 \\
\hline $\mathrm{S} \times \mathrm{P} \times \mathrm{C}$ & 2 & 127,60 & 58 & 4,02 & 31,75 & .000 & .52 \\
\hline $\mathrm{S} \times \mathrm{P} \times \mathrm{H}$ & 2 & 87,50 & 58 & 2,02 & 43,36 & .000 & .60 \\
\hline $\mathrm{S} \times \mathrm{P} \times \dot{\mathrm{E}}$ & 2 & 0,64 & 58 & 0,51 & 1,27 & .290 & .04 \\
\hline $\mathrm{S} \times \mathrm{C} \times \mathrm{H}$ & 2 & 4,37 & 58 & 1,49 & 2,92 & .062 & .09 \\
\hline $\mathrm{S} \times \mathrm{C} \times \dot{\mathrm{E}}$ & 2 & 1,69 & 58 & 0,48 & 3,55 & .035 & .11 \\
\hline $\mathrm{S} \times \mathrm{H} \times \overline{\mathrm{E}}$ & 2 & 7,01 & 58 & 0,42 & 16,65 & .000 & .36 \\
\hline $\mathrm{P} \times \mathrm{C} \times \hat{\mathrm{E}}$ & 1 & 1,11 & 29 & 0,60 & 1,84 & .185 & .06 \\
\hline $\mathrm{P} \times \mathrm{C} \times \mathrm{H}$ & 1 & 204,00 & 29 & 1,71 & 119,12 & .000 & .80 \\
\hline $\mathrm{P} \times \mathrm{H} \times \mathrm{E}$ & 1 & 5,38 & 29 & 0,74 & 7,24 & .012 & .20 \\
\hline $\mathrm{C} \times \mathrm{H} \times \dot{\mathrm{E}}$ & 1 & 0,80 & 29 & 0,44 & 1,83 & .186 & .06 \\
\hline
\end{tabular}

Une ANOVA suivant le plan factoriel Problème $x$ Classe $\times$ Habitude $\times$ Équipe d'Éducation Physique et Sportive a été menée sur les données provenant de la régulation par la sanction pour chaque population. Le tableau 4 présente les principaux résultats de l'ANOVA menée sur les deux groupes.

De manière générale, les jugements de pertinence de régulation par la sanction des titulaires $(M=5,33$; $S D=0,16)$ sont moins élevés que ceux des stagiaires $(M=7,17 ; S D=0,22)$. Le tableau 5 présente les principales moyennes et écart-types pour les deux populations. Les quatre variables intra-sujets ont eu un effet significatif dans les deux populations. Que ce soit chez les stagiaires ou les titulaires, la sanction a été jugée plus légitime dans des situations de perturbation, en sixième, pour des élèves habitués à ce type de comportements, et lorsque l'équipe d'EPS a pour habitude d'adopter cette réaction.

La figure 2 présente le patron d'intégration du problème rencontré, de la classe et de l'habitude de l'élève à adopter un type de comportement déviant, sur le jugement de pertinence de la régulation par la sanction dans les deux groupes. La figure 2a concerne la population des stagiaires. Quelle que soit l'habitude de l'élève à adopter le comportement déviant et le problème, le
Tableau 3. Moyennes marginales et écart-types de chaque facteur et l'Interaction stratégie $\times$ problème issus des différentes populations.

\begin{tabular}{|c|c|c|c|c|}
\hline \multirow[b]{2}{*}{ Facteurs } & \multicolumn{2}{|c|}{ Stagiaires } & \multicolumn{2}{|c|}{ Titulaires } \\
\hline & $M$ & $S D$ & $M$ & $S D$ \\
\hline \multicolumn{5}{|l|}{ Stratégie } \\
\hline Sanction & 7,17 & 0,22 & 5,33 & 0,16 \\
\hline Négociation & 1,83 & 0,16 & 1,84 & 0,15 \\
\hline Compréhension & 0,87 & 0,20 & 2,16 & 0,17 \\
\hline \multicolumn{5}{|l|}{ Problème } \\
\hline Perturbation & 3,13 & 0,13 & 3,30 & 0,09 \\
\hline Refus & 3,45 & 0,20 & 2,92 & 0,17 \\
\hline \multicolumn{5}{|l|}{ Classe } \\
\hline Sixième & 3,17 & 0,12 & 2,89 & 0,12 \\
\hline Terminal & 3,41 & 0,19 & 3,33 & 0,13 \\
\hline \multicolumn{5}{|l|}{ Habitude } \\
\hline Habitué & 3,11 & 0,12 & 2,93 & 0,12 \\
\hline Non Habitué & 3,47 & 0,19 & 3,29 & 0,12 \\
\hline \multicolumn{5}{|l|}{ Équipe D'ÉPS } \\
\hline Non-Utilisation & 2,93 & 0,14 & 2,81 & 0,10 \\
\hline Utilisation & 3,65 & 0,16 & 3,40 & 0,13 \\
\hline \multicolumn{5}{|l|}{ Stratégie $\times$ Problème } \\
\hline Sanction $\times$ Perturbation & 7,82 & 0,20 & 7,80 & 0,15 \\
\hline Sanction $\times$ Refus & 6,52 & 0,29 & 2,26 & 0,23 \\
\hline Négociation $\times$ Perturbation & 1,07 & 0,19 & 1,17 & 0,12 \\
\hline Négociation $\times$ Refus & 2,60 & 0,25 & 2,51 & 0,22 \\
\hline Compréhension $\times$ Perturbation & 0,50 & 0,16 & 0,94 & 0,11 \\
\hline Compréhension $\times$ Refus & 1,25 & 0,28 & 3,38 & 0,26 \\
\hline
\end{tabular}

jugement de pertinence de la régulation par la sanction a toujours été élevé pour une classe de sixième. Les tests de post hoc de Tukey ont confirmé ces non-différences de jugement $\left(p_{\mathrm{s}}>.05\right)$.

La figure $2 \mathrm{~b}$ concerne la population des titulaires. Ils différencient leur jugement de pertinence de la régulation par la sanction en fonction du problème et de l'habitude. Ils jugent plus pertinent la stratégie de sanction pour la perturbation et lorsque l'élève est un habitué de ce type de comportement déviant. Les tests post hoc de Tukey ont confirmé la significativité de ces différences de jugement $\left(p_{\mathrm{s}}<.001\right)$.

\section{Discussion}

Notre étude visait à mettre en évidence les différences entre enseignants et stagiaires en ce qui concerne leur façon de combiner mentalement différentes informations pour estimer la pertinence de la régulation des comportements déviants durant une leçon en Éducation Physique et Sportive. La régulation des comportements déviants est une question essentielle pour les enseignements en Éducation Physique et Sportive car maintenir l'ordre et la sécurité est l'une de leurs préoccupations majeures (Nault \& Lacourse, 2008). 

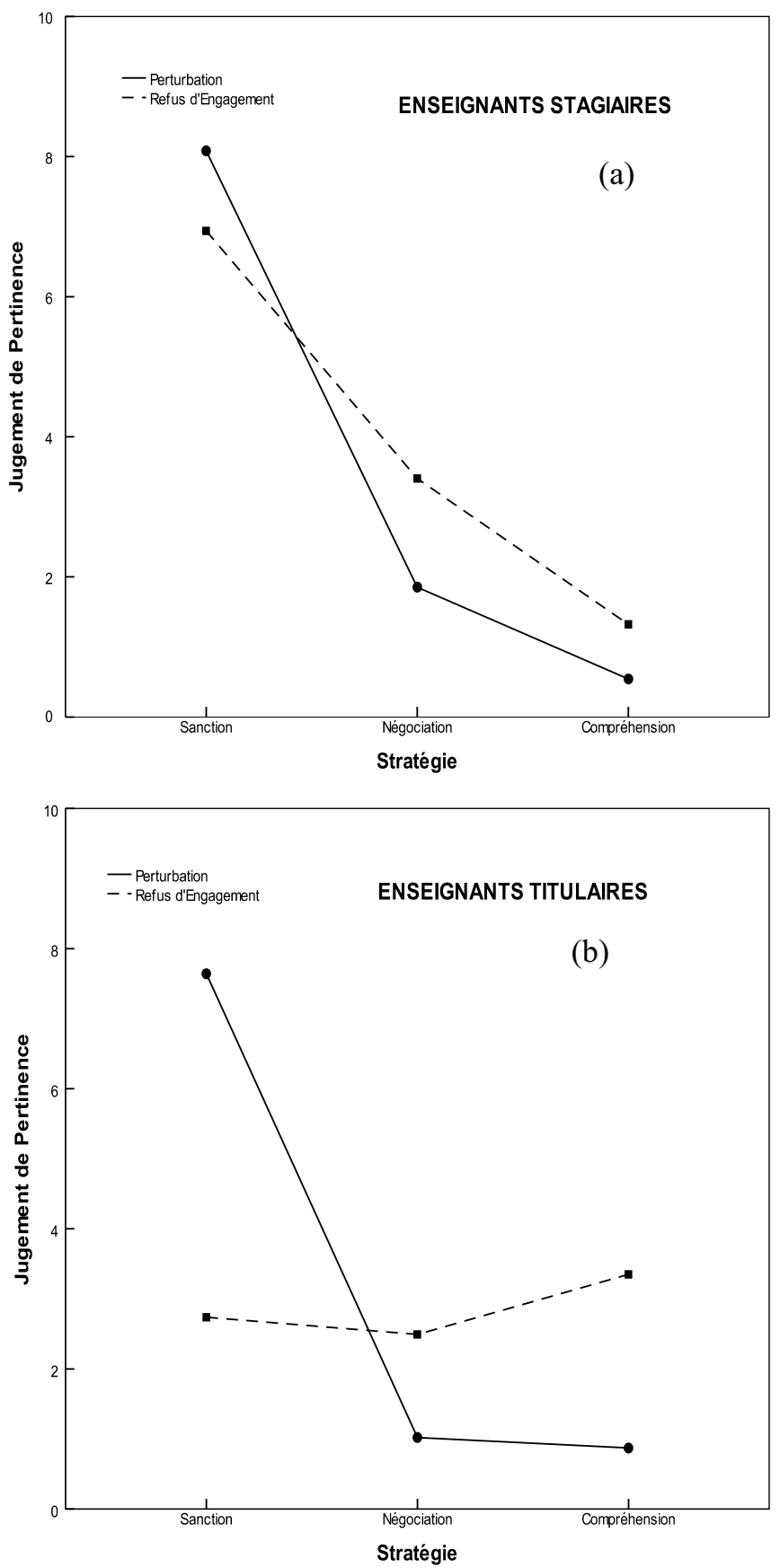

Figure 1. Effet combiné de la stratégie et du problème rencontré sur le jugement de pertinence de la régulation dans les populations. a : stagiaires; $\mathrm{b}$ : titulaires.

La première hypothèse était que les participants prendraient en considération le type de stratégie de régulation, la nature du problème auquel l'enseignant est confronté, la classe de l'élève, l'habitude de l'élève à adopter ce type de comportement déviant, et l'emploi de ce type de stratégie par l'équipe d'Éducation Physique et Sportive pour juger la pertinence de la régulation des comportements déviants. Cette hypothèse est confirmée pour trois des cinq facteurs : Classe, Habitude et Équipe. En ce qui concerne le facteur Stratégie, les participants n'ont pas différencié leurs jugements de pertinence de régulation entre les stratégies
Tableau 4. Principaux résultats des ANOVAs effectuées sur les données des stagiaires et des titulaires pour la stratégie par sanction.

\begin{tabular}{|c|c|c|c|c|c|c|c|}
\hline \multirow[b]{2}{*}{ Facteurs } & \multicolumn{2}{|c|}{ Effet } & \multicolumn{2}{|c|}{ Erreur } & \multirow[b]{2}{*}{$F$} & & \multirow[b]{2}{*}{$\eta_{p}^{2}$} \\
\hline & $d f$ & $M S$ & $d f$ & $M S$ & & & \\
\hline \multicolumn{8}{|l|}{ Stagiaires } \\
\hline Problème $(\mathrm{P})$ & 1 & 164,06 & 23 & 4,56 & 35,94 & .000 & .61 \\
\hline Classe (C) & 1 & 265,00 & 23 & 3,48 & 77,34 & .000 & .77 \\
\hline Habitude $(\mathrm{H})$ & 1 & 164,6 & 23 & 2,13 & 77,01 & .000 & .77 \\
\hline Équipe d'ÉPS (É) & 1 & 48,88 & 23 & 0,62 & 79,25 & .000 & .77 \\
\hline $\mathrm{P} \times \mathrm{C}$ & 1 & 196,94 & 23 & 4,05 & 48,64 & .000 & .68 \\
\hline $\mathrm{P} \times \mathrm{H}$ & 1 & 10,34 & 23 & 1,87 & 5,53 & .028 & .19 \\
\hline $\mathrm{P} \times \mathrm{E}$ & 1 & 0,02 & 23 & 0,40 & 0,06 & .812 & .00 \\
\hline $\mathrm{C} \times \mathrm{H}$ & 1 & 9,06 & 23 & 3,33 & 2,73 & .112 & .11 \\
\hline $\mathrm{C} \times \mathrm{E}^{\prime}$ & 1 & 0,02 & 23 & 0,56 & 0,04 & .841 & .00 \\
\hline $\mathrm{H} \times \hat{\mathrm{E}}$ & 1 & 0,21 & 23 & 0,57 & 0,37 & .549 & .02 \\
\hline $\mathrm{P} \times \mathrm{C} \times \mathrm{H}$ & 1 & 32,08 & 23 & 2,01 & 15,96 & .001 & .41 \\
\hline $\mathrm{P} \times \mathrm{C} \times \dot{\mathrm{E}}$ & 1 & 0,75 & 23 & 0,44 & 1,72 & .203 & .07 \\
\hline $\mathrm{P} \times \mathrm{H} \times \mathrm{E}$ & 1 & 0,02 & 23 & 0,91 & 0,03 & .874 & .00 \\
\hline $\mathrm{C} \times \mathrm{H} \times \mathrm{E}$ & 1 & 0,31 & 23 & 0,39 & 0,80 & .379 & .03 \\
\hline $\mathrm{P} \times \mathrm{C} \times \mathrm{H} \times \mathrm{E}^{\prime}$ & 1 & 0,75 & 23 & 0,86 & 0,87 & .360 & .04 \\
\hline
\end{tabular}

Titulaires

\begin{tabular}{lrrrrrrr} 
Problème (P) & 1 & 2925,47 & 23 & 5,38 & 543,95 & .000 & .95 \\
Classe (C) & 1 & 117,02 & 23 & 5,42 & 21,59 & .000 & .43 \\
Habitude (H) & 1 & 365,75 & 23 & 1,16 & 314,88 & .000 & .92 \\
Équipe d'ÉPS (É) & 1 & 74,42 & 23 & 1,52 & 49,03 & .000 & .63 \\
$\mathrm{P} \times \mathrm{C}$ & 1 & 59,50 & 23 & 5,75 & 10,35 & .003 & .26 \\
$\mathrm{P} \times \mathrm{H}$ & 1 & 14,35 & 23 & 2,78 & 5,16 & .031 & .15 \\
$\mathrm{P} \times \mathrm{E}$ & 1 & 5,85 & 23 & 0,78 & 7,51 & .010 & .21 \\
$\mathrm{C} \times \mathrm{H}$ & 1 & 39,10 & 23 & 1,74 & 22,42 & .000 & .44 \\
$\mathrm{C} \times \mathrm{E}$ & 1 & 0,17 & 23 & 0,64 & 0,26 & .611 & .01 \\
$\mathrm{H} \times \hat{\mathrm{E}}$ & 1 & 3,50 & 23 & 0,82 & 4,24 & .048 & .13 \\
$\mathrm{P} \times \mathrm{C} \times \mathrm{H}$ & 1 & 46,25 & 23 & 2,12 & 21,83 & .000 & .43 \\
$\mathrm{P} \times \mathrm{C} \times \mathrm{E}$ & 1 & 0,02 & 23 & 0,56 & 0,03 & .856 & .00 \\
$\mathrm{P} \times \mathrm{H} \times \hat{\mathrm{E}}$ & 1 & 22,97 & 23 & 1,57 & 14,65 & .001 & .34 \\
$\mathrm{C} \times \mathrm{H} \times \dot{\mathrm{E}}$ & 1 & 0,25 & 23 & 0,46 & 0,54 & .467 & .02 \\
$\mathrm{P} \times \mathrm{C} \times \mathrm{H} \times \hat{\mathrm{E}}$ & 1 & 2,27 & 23 & 0,62 & 3,67 & .065 & .11 \\
\hline
\end{tabular}

de négociation et de compréhension. En revanche, ils les ont différenciés entre, d'une part, la sanction et, d'autre part, la négociation et la compréhension. Ce résultat confirme l'existence de deux principaux modes de régulation chez les enseignants (Grimault-Leprince, 2011) avec d'un côté, le formalisme (lié à la sanction) et de l'autre, le pragmatisme (lié à la négociation et à la compréhension). Toutefois, la divergence entre le pragmatisme utilitariste et le pragmatisme compréhensif mise en évidence par GrimaultLeprince (2011) n'a pas été démontrée dans la présente étude. Le facteur problème n'a pas été significatif dans l'ANOVA menée sur l'ensemble des données pour renseigner la pertinence de la régulation. Les participants ont globalement jugé la pertinence de la régulation de la même manière quel que soit le problème rencontré. 
Tableau 5. Moyennes marginales et écart-types de chaque facteur issus des populations pour la stratégie de régulation par sanction.

\begin{tabular}{|c|c|c|c|c|}
\hline \multirow[b]{3}{*}{ Facteurs } & \multicolumn{4}{|c|}{ Domination } \\
\hline & \multicolumn{2}{|c|}{ Stagiaires } & \multicolumn{2}{|c|}{ Titulaires } \\
\hline & $M$ & $S D$ & $M$ & $S D$ \\
\hline \multicolumn{5}{|l|}{ Problème } \\
\hline Perturbation & 7,82 & 0,16 & 7,80 & 0,15 \\
\hline Refus & 6,52 & 0,18 & 2,86 & 0,22 \\
\hline \multicolumn{5}{|l|}{ Classe } \\
\hline Sixième & 8,00 & 0,18 & 5,82 & 0,20 \\
\hline Terminal & 6,34 & 0,29 & 4,84 & 0,19 \\
\hline \multicolumn{5}{|l|}{ Habitude } \\
\hline Habitué & 7,82 & 0,23 & 6,20 & 0,18 \\
\hline Non Habitué & 6,52 & 0,24 & 4,46 & 0,16 \\
\hline \multicolumn{5}{|l|}{ Équipe D'ÉPS } \\
\hline Non-Utilisation & 6,81 & 0,24 & 4,94 & 0,16 \\
\hline Utilisation & 7,53 & 0,22 & 5,72 & 0,18 \\
\hline
\end{tabular}

En revanche, la régulation a été jugée plus pertinente quand les enseignants utilisent la sanction, quand l'élève se trouve en classe de sixième, quand il est un habitué de ce type de comportement déviant et quand l'équipe d'Éducation Physique et Sportive à laquelle l'enseignant appartient utilise cette stratégie de régulation. Elle a été jugée moins pertinente quand les enseignants emploient la négociation ou la compréhension, quand l'élève se trouve en terminale, quand il n'est pas un habitué des comportements déviants et quand l'équipe d'Éducation Physique et Sportive à laquelle l'enseignant appartient n'utilise pas cette stratégie de régulation. Ces résultats témoignent d'une certaine invariance dans la prise en compte des facteurs justifiant une régulation d'un comportement déviant. D'un point de vue épistémique, on a une croyance en la pertinence d'une régulation reposant sur des justifications solides: si l'enseignant utilise la sanction, si l'élève est jeune, récidiviste dans ses comportements déviants et si l'équipe enseignante adopte cette stratégie répressive.

Notre deuxième hypothèse était qu'une différence de jugement de pertinence de la régulation devrait être trouvée entre les titulaires et les stagiaires. Cette hypothèse a été confirmée. Les enseignants titulaires et les stagiaires se distinguent donc dans leur manière de juger la pertinence de la régulation des comportements déviants en Éducation Physique et Sportive en fonction de la stratégie mise en place et de la nature du problème auquel ils sont confrontés. Ce résultat confirme les conclusions des recherches sur l'existence d'une différence d'efficacité perçue de gestion de classe entre enseignants titulaires et stagiaires (De la Torre Cruz et Arias, 2007) ou sur l'impact de l'expérience sur l'efficacité perçue de la régulation des comportements déviants (e.g., Tsouloupas, Carson, \& Matthews, 2014). Ils soulignent également l'importance des processus cognitifs pour la formation.
Les titulaires estiment différemment la pertinence de la régulation en fonction de la nature du problème rencontré et de la stratégie mise en place. Face à un problème de perturbation, ils jugent pertinent de la réguler par la sanction. Face à un problème de refus d'engagement dans l'activité, ils considèrent les régulations par sanction, négociation et compréhension toutes aussi pertinentes les unes que les autres. Ces résultats confirment ceux de Kearney (1988) qui soulignent la capacité cognitive des enseignants titulaires à utiliser différentes stratégies de régulation en fonction du problème rencontré. Selon l'auteur, par leur activité et leurs interactions sociales, les titulaires développeraient des processus cognitifs bien intégrés et sophistiqués qui leur permettraient de choisir la bonne régulation face à des comportements déviants présentés a priori.

À l'inverse, pour les stagiaires, quel que soit la nature du problème rencontré, la régulation par la sanction a été jugée la plus pertinente, suivie de celle par la négociation et de celle par la compréhension. Ce résultat confirme de nombreuses conclusions issues de la littérature (Almog \& Shechtman, 2007; Dervaux et al., 2004; Goyette et al., 2000). Les novices se centreraient sur eux-même, sur leurs préoccupations, c'est-à-dire qu'ils se concentreraient sur leurs pratiques pédagogiques qui renforceraient leur image personnelle (Desbiens et al., 2011). À travers cette stratégie de sanction, les novices pourraient avoir l'impression d'un contrôle direct de la classe, ce qui influencerait positivement leur motivation et leur investissement (Kyriacou, Avramidis, Hoie, Stephen, \& Hultgren, 2007). Même s'ils sont sensibles à la négociation, ils jugent une régulation par la négociation peu pertinente alors qu'une pédagogie interactive est recommandée pour développer l'autonomie, la responsabilité et l'apprentissage de l'élève (Reeve, 2002). Quant à la régulation par la compréhension, elle s'appuie sur une connaissance approfondie de l'adolescence (Grimault-Leprince, 2011) et on peut supposer qu'elle demande une certaine confiance en soi renforcée par l'expérience professionnelle (Kyriacou et al., 2007). Cette expérience va se traduire par une forme de «resocialisation » organisée sur le principe de l'intégration par l'élève des règles de conduite liées au maintien de l'ordre scolaire. L'enjeu est de sensibiliser les élèves à une forme de restauration du sens de la sanction qui ne se limite pas au rappel de la loi scolaire. Ceci est la condition d'entrée dans les apprentissages et pourrait expliquer que les stagiaires ne soient pas encore en mesure d'envisager ce type de régulation.

Cette distinction entre enseignants stagiaires et titulaires est soutenue par l'identification de trois types de règles cognitives présentées par Anderson (1996). Une application de ces règles cognitives dans le domaine sportif précise que la forme des courbes obtenues sur les graphiques renvoie à une règle cognitive particulière (pour plus d'explications, voir Rulence-Pâques, Fruchart, Dru, \& Mullet, 2005). La manière dont les participants ont combiné les informations pour juger la pertinence de la régulation par la sanction a produit des règles cognitives simples. Face à un problème de perturbation, une règle 


\section{(a) ENSEIGNANTS STAGIAIRES}
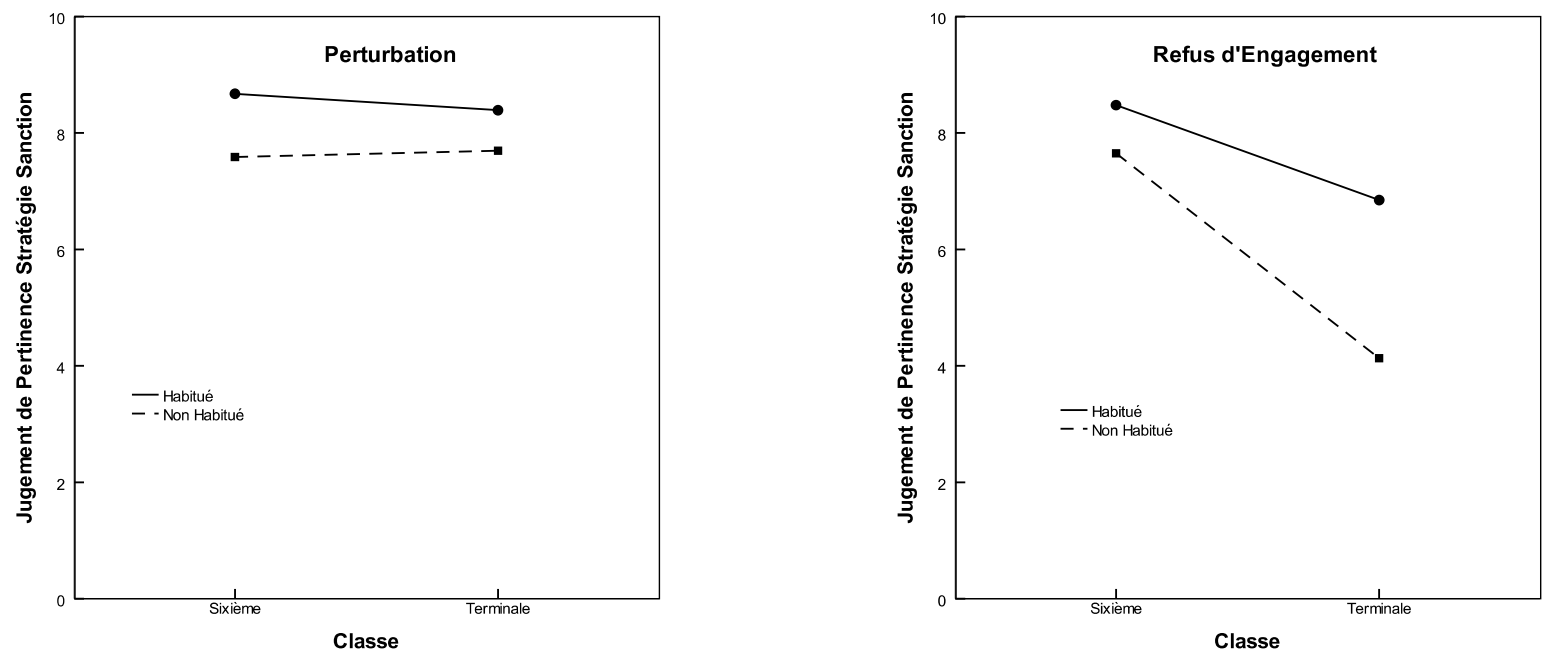

(b) ENSEIGNANTS TITULAIRES
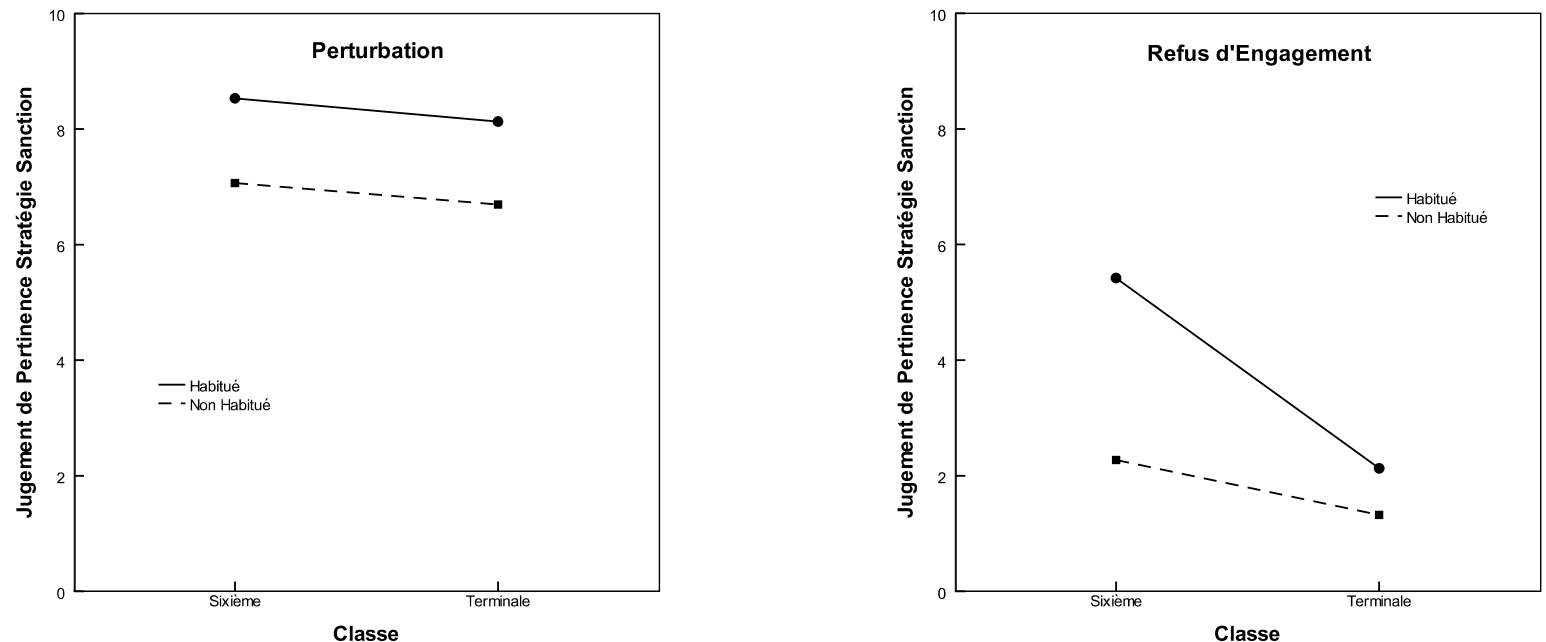

Figure 2. Effet combiné du problème rencontré, de la classe et de l'habitude de l'élève à adopter le type comportement déviant, sur le jugement de pertinence de la stratégie de sanction dans les deux groupes. a : stagiaires; b : titulaires.

additive (caractérisée par des courbes parallèles) a été identifiée pour les enseignants comme pour les stagiaires. Cette règle traduit que la sanction sera jugée forte si l'élève est jeune et ceci, d'autant plus qu'il réitère son comportement perturbateur. Par contre, face à un refus d'engagement dans l'activité, les titulaires et les stagiaires emploient des règles cognitives opposées. Une règle conjonctive est utilisée par les stagiaires (caractérisée par des courbes formant un éventail ouvert à droite) traduisant l'importance accordée au comportement habituel des élèves plus âgés. À l'inverse, une règle disjonctive est utilisée par les titulaires (caractérisée par des courbes formant un éventail ouvert à gauche) traduisant l'importance accordée au comportement habituel des élèves les plus jeunes. Ainsi, l'étude des règles cognitives déjà appliquée dans de nombreux domaines en psychologie (pour une revue, Anderson, 2008) permet de comprendre la différence entre enseignants titulaires et stagiaires, en ce qui concerne la pertinence de la régulation par la sanction des comportements déviants en Éducation Physique et Sportive. Comprendre la manière dont les enseignants titulaires ou stagiaires intègrent cognitivement diverses informations issues d'un contexte standardisé et implémenter la théorie fonctionnelle de la cognition (Anderson, 1996) est une approche précieuse pour renseigner la question de la gestion de classe en Éducation Physique et Sportive.

Notre étude présente toutefois quelques limites. La première limite concerne l'approche théorique et méthodologique de notre étude. Contrairement à l'approche de la 
cognition située (e.g., Vors \& Gal-Petitfaux, 2014), nous ne pouvons prendre en considération l'expérience des enseignants et des élèves, la manière dont ils vivent les situations de régulation des comportements déviants. Sa caractéristique «objectiviste» ne laisse aucune place à l'imprévisibilité (e.g., Méard et al., 2008). Plutôt que de mettre en opposition l'approche de l'action située et notre approche cognitiviste, nous pourrions envisager de les mettre conjointement à profit avec un minimum de cohérence pour comprendre le phénomène de régulation dans le domaine de l'intervention (Desbiens, 2010). Sur le plan méthodologique, l'aspect «caricatural» de nos scénarios composés de facteurs trop tranchés peut être discuté, avec un manque de considération de niveau intermédiaire pour les différents facteurs. Par exemple, l'équipe d'Éducation Physique et Sportive aurait pu employer «de temps en temps» cette régulation. Une des principales limites de cette méthodologie est de présenter un ensemble limité de facteurs susceptibles d'impacter le jugement. Toutefois, croiser trop de variables à plusieurs degrés risquerait d'engendrer une lassitude et/ ou une surcharge cognitive chez les pratiquants (Anderson, 2008). D'autres éléments sont susceptibles d'influer sur le jugement de pertinence d'une stratégie de régulation, au même titre que les cinq facteurs retenus.

En ce sens, une deuxième limite concerne le choix des variables de cette étude. Selon le regard porté aux différentes approches scientifiques, d'autres facteurs auraient pu être considérés. Des travaux appliquant l'approche située soulignent l'importance d'autres éléments du contexte: l'histoire de la classe (phénomène d'accumulation ou de «goutte d'eau»), la dynamique de classe, la singularité des enseignants, l'ambiance de l'établissement, la forme de l'activité, la co-construction des règles entre les enseignants et élèves, l'Activité Physique Sportive et Artistique support (e.g., Flavier et al., 2002 ; Guérin et al., 2005 ; Méard et al., 2008; Vors \& Gal-Petitfaux, 2014). En se référant à l'approche comportementaliste (e.g., Turcotte et al., 2008), d'autres comportements déviants auraient pu être investis tels les retards, le fait de ne pas être en possession de sa tenue d'Éducation Physique et Sportive, le fait d'être grossier. Nous pourrions tester ces différentes variables dans de futures investigations.

Une troisième limite concerne la dimension non écologique de cette approche scientifique. En effet, on pourrait se demander si ce type de stratégie opère réellement en situation. Pour répondre à ce point, il pourrait être envisagé une comparaison directe entre des schémas de jugement dérivés de l'étude de laboratoire (à partir de questionnaires) et des schémas de jugement inférés d'observations en situations réelles (vidéo de terrain). Une compatibilité raisonnable a déjà été validée dans le domaine de la remise en jeu en sport collectif (Fruchart, Rulence-Pâques, \& Mullet, 2007). Les schémas de jugement relèvent de la psychologie cognitive où l'accent est mis sur l'intégration de l'information. Lorsqu'ils sont inférés d'observations en situations réelles, ces jugements relèvent du processus d'interaction entre l'individu et son environnement (physique, matériel et social) pour comprendre l'activité humaine. De manière similaire à l'étude validée dans le domaine de la remise en jeu en sport collectif (Fruchart et al., 2007), des vidéos de terrain pourraient être utilisées et témoigneraient des interactions prises en compte justifiant le mode de régulation utilisé. Des entretiens d'autoconfrontation (Theureau, 2006) pourraient alors être menés auprès des participants afin de récupérer leur expérience.

Une quatrième limite se rapporte aux participants. La question de l'expertise mériterait d'être approfondie avec la prise en considération des années d'ancienneté des enseignants et en mobilisant des participants plus expérimentés que ceux concernés par notre étude (e.g., Visioli \& Ria, 2010). Enfin, la question du genre aurait pu être posée puisque des différences entre les hommes et les femmes ont été identifiées chez les enseignants dans leur réaction aux comportements déviants dans le domaine de l'Éducation Physique et Sportive (e.g., Desbiens et al., 2011; Lentillon, 2007; Couchot-Schiex, Cogérino, \& Coltice, 2009): la pertinence de la régulation va-t-elle être jugée de la même manière par des participants masculins et des participants féminins? La régulation décidée sera-t-elle identique selon que l'élève soit une fille ou un garçon? L'ensemble de ces limites pourront être prises en considération dans des recherches ultérieures.

Les résultats de cette recherche pourraient avoir des implications dans la formation des futurs enseignants en Éducation Physique et Sportive. Des histoires semblables manipulant des situations pédagogiques problématiques variées pourraient être proposées telles que l'ont fait Chasseigne, Giraudeau, Lafon et Mullet (2011) en psychologie de l'éducation ou l'ont présenté Fruchart, Dracon, Pâques et Dru (2009) en psychologie du sport. De manière identique, ces mêmes situations pourraient être soumises aux élèves afin de comparer leur degré d'accord avec le type de régulation décidé par l'enseignant. Les réponses recueillies pourraient constituer le point de départ d'une réflexion sur les pratiques pédagogiques aidant à réduire les comportements déviants et entraînant la transmission des valeurs de citoyenneté et de respect des autres.

\section{Références}

Almog, O., \& Shechtman, Z. (2007). Teachers' democratic and efficacy beliefs and styles of coping with behavioural problems of pupils with special needs. European Journal of Special Needs Education, 22, 115-129.

Anderson, N.H. (1996). A functional theory of cognition. Mahwah, NJ: Erlbaum.

Anderson, N.H. (2008). Unified social cognition. New York: Psychology Press.

Ayme, S., Ferrand, C., \& Cogérino, G. (2011). Comportements déviants des élèves et émotion de colère des enseignants en EPS. Carrefour de l'Éducation, 32, 213-227.

Barker, D., \& Annerstedt, C. (2014). Managing physical education lessons: an interactional approach. Sport Education and Society. doi: 10.1080/13573322.2014.969229. 
Bandura, A. (1997). Self-efficacy: The exercise of control. New York: W.H. Freeman and Company.

Chasseigne, G., Giraudeau, C., Lafon, P., \& Mullet, E. (2011). Improving students' ability to intuitively resistance from magnitude of current and potential difference information: A functional learning approach. European Journal of Psychology Education, 26, 1-19. doi: 10.1007/s10212-010-0048-z.

Couchot-Schiex, S., Cogérino, G., \& Coltice, M. (2009). Professeurs stagiaires en EPS face à l'enseignement en mixité. Carrefours de l'Éducation, 27, 169-182.

Cruz, A. (2006). Class management on in-service physical Education student teachers. Journal of Physical Education et Recreation, 11, 14-23.

Day, C., Sammons, P., Stobart, G., Kingston, A., \& Gu, Q. (2007). Teachers matter: Connecting lives, work and effectiveness. Maidenhead, England: Open University Press.

De la Torre Cruz, M.J., \& Arias, P.F.C. (2007). Comparative analysis of expectancies of efficacy in in-service and prospective teachers. Teaching and Teacher Education, 23, 641-652.

Dervaux, P., Carlier, G., \& Gérard, G. (2004). Comment des enseignants stagiaires déclarent-ils gérer six situations d'indiscipline? In M. Locquet \& Y. Léziart (dir.), Cultures sportives et artistiques: formalisation des savoirs professionnels. Pratiques, formations, recherches (pp. 229-232). Rennes: Université de Haute Bretagne, Rennes II.

Desbiens, J.-F. (2010). L'action située: critique mesurée d'une approche novatrice de l'apprentissage. eJRIEPS, 10, $4-27$.

Desbiens, J.-F., Turcotte, S., Spallanzani, C., Roy, M., Brunelle, J.-P., Tourigny, J.-S., \& Lanoue, S. (2008). Une analyse des comportements perturbateurs survenus durant des cours d'éducation physique et à la santé enseignés par des stagiaires. STAPS, 81, 73-88.

Desbiens, A., Turcotte, S., Spallanzani, C., Roy, M., Tourigny, J.-S., \& Lanoue, S. (2011). Comment des stagiaires en enseignement de l'éducation physique et à la santé (EPSa) réagissent-ils face à l'indiscipline de leurs élèves? Science $\mathscr{E}$ Motricité, 73, 39-54.

Fernandez-Balboa, J.M. (1991). Beliefs, interactive thoughts, and actions of physical Education students teachers regarding pupil misbehaviors. Journal of Teaching in Physical Education, 11, 59-78.

Flavier, E., Bertone, S., Méard, J., \& Durand, M. (2002). Les préoccupations des professeurs d'éducation physique lors de la genèse et la régulation des conflits en classe. Revue Française de Pédagogie, 139, 107-119.

Fruchart, E., \& Rulence-Pâques, P. (2014). Condoning aggressive behaviour in sport: A comparison between professional handball players, amateur players, and lay people. Psicológica, 35, 585-600.

Fruchart, E., Rulence-Pâques, P., \& Mullet, E. (2007). Ecological validity test of laboratory studies on information integration. Teorie 83 Modelli, 12/1-12, 281-288.

Fruchart, E., Dracon, N., Pâques, P., \& Dru, V. (2009). Applications de la théorie fonctionnelle de la cognition III. In B. Cadet \& G. Chasseigne (Eds.), Jugement et prises de décision. Bruxelles: De Boeck.

Fruchart, E., Pâques, P., Dru, V., \& Mullet, E. (2010). Decisionmaking in basketball and handball games: a developmental perspective. European Review of Applied Psychology, 60, 2734. doi: 10.1016/j.erap.2009.10.003.

Goyette, R., Doré, R., \& Dion, E. (2000). Pupils' misbehaviour and the reactions and causal attributions of physical Education student teachers: a sequential analysis. Journal of Teaching in Physical Education, 20, 3-14.
Grimault-Leprince, A. (2011). La gestion de la classe par les enseignants de collège. Formalisme versus pragmatisme. Carrefours de l'Éducation, 31, 217-235. doi:10.3917/ cdle.031.0217.

Guérin, J., Testevuide, S., \& Roncin, C. (2005). Les effets des «situations-jeu » en tennis de table sur l'activité d'un élève en cours d'éducation physique. STAPS, 69, 105-118.

Hastie, P.A., \& Siedentop. D. (2006). The classroom ecology paradigm. In D. Kirk, D. MacDonald, \& M. O'Sullivan (Eds.), Handbook of Physical Education (pp. 214-225). London: Sage.

Jelen, N. (2014). L'influence des collègues d'éducation physique et sportive (EPS) dans l'usage des savoirs pour réguler les comportements violents en EPS. International Journal of Violence and Schools, 14, 31-43.

Kearney, P. (1988). Experienced and prospective teachers' compliance-gaining message selections on common student misbehaviors. Communication Education, 37, 150-164. doi: 10.1080/03634528809378712.

Kulinna, P.H., Cothran, D.J., \& Regualos, R. (2003). Development of an instrument to measure student disruptive behaviors. Measurement in Physical Education and Exercise Science, 7, 25-41.

Kulinna, P.H., Cothran, D.J., \& Regualos, R. (2006). Teachers' reports of students misbehavior in physical education. Research Quarterly for Exercise and Sport, 77, 32-40.

Kyriacou, C., Avramidis, E., Hoie, H., Stephen, P., \& Hultgren, (2007). The development of student teachers' views on pupil misbehaviour during an initial teacher training programme in England and Norway. Journal of Education for Teaching, 33, 293-307.

Lavay, B., Henderson, H., French, R., \& Guthrie, S. (2012). Behavior management instructional practices and content of college/university physical education teacher education (PETE) programs. Physical Education 6 Sport Pedagogy, 17, 195-210. doi: 10.1080/17408989.2010.548063.

Lentillon, V. (2007). Notes et perceptions de privation chez les élèves en éducation physique sportive: variations selon leur sexe et leur orientation de genre. Les Cahiers Internationaux de Psychologie Sociale, 75-76, 79-91.

Lewis, T., Romi, S., Qui, X., \& Katz, Y.J. (2005). Teachers' classroom discipline and student misbehavior in Australia, China and Israel. Teaching and Teacher Education, 21, 729741.

Méard, J., Bertone, S., \& Flavier, E. (2008). How second-grade students internalize rules during teacher-student transactions: A case study. British Journal of Educational Psychology, 78, 395-410. doi: 10.1348/000709907X264141.

Morin, J., \& Battalio, R. (2004). Construing misbehavior: the efficacy connection in responding to misbehavior. Journal of Positive Behaviour Interventions, 6, 251-254.

Nault, T., \& Lacourse, F. (2008). La gestion de classe. Une compétence à développer. Anjou: Les Éditions CEC.

Petiot, O., Visioli, J., \& Desbiens, J.-F. (2015). Perceptions d'enseignants du secondaire concernant leurs inducteurs émotionnels en situation de classe. Revue Française de Pédagogie, 193, 41-55. doi: 10.4000/rfp.4886.

Piaget, J. (1970). The science of education and the psychology of the child. New York: Orion Press.

Reeve, J. (2002). Self-determination theory applied to educational settings. In E.L. Deci \& R.M. Ryan (dir.), Handbook of self-determination research (pp. 183-204). Rochester N.-Y.: The University Press of Rochester.

Rulence-Pâques, P., \& Mullet, E. (1998). Area judgment from width and height information: the case of the rectangle. Journal of Experimental Child Psychology, 69, 22-48. 
Rulence-Pâques, P., Fruchart, E., Dru, V., \& Mullet, E. (2005). Cognitive algebra in sport decision-making. Theory and Decision, 58, 387-406. doi: 10.1007/s11238-005-3890-8.

Supaporn, S., Doods, P., \& Griffin, L. (2003). An ecological analysis of middle school misbehaviour through student and teacher perspectives. Journal of Teaching in Physical Education, 22, 328-349.

Theureau, J. (2006). Cours d'action: méthode développée. Toulouse : Octarès.

Tsouloupas, C.N., Carson, R.L., \& MacGregor, S.K. (2014). The development of High School Teachers' Efficacity in Handling Student Mibehavior (TEHSM). The Journal of Educational Research, 107, 230-240. doi: 10.1080/00220671.2013.788992.

Tsouloupas, C.N., Carson, R.L., \& Matthews, R.A. (2014). Personal and school cultural factors associated with the perceptions of teachers' efficacy in handling student misbehavior. Psychology in the Schools, 51, 164-180. doi: 10.1002/ pits.21739.

Turcotte, S., Desbiens, J.F., Spallanzani, C., Roy, M., Brunelle, J.P., \& Tourigny, J.S. (2008). Portrait des comportements perturbateurs adoptés par des élèves du niveau primaire en éducation physique et à la santé. eJRIEPS, 213, 57-77.

Varela, F.J. (1989). Invitations aux sciences cognitives. Paris: Seuil.

Visioli, J., \& Ria, L. (2010). L'expertise des enseignants d'EPS. Movement et Sport Sciences, 71, 3-19.

Vors, O., \& Gal-Petitfaux, N. (2014). Relation between students' involvement and teacher management strategies in French 'difficult' classrooms. Physical Education and Sport Pedagogy. doi: 10.1080/17408989.2014.882889.

Citation de l'article : Fruchart É \& Rulence-Pâques P (2018) Juger la pertinence de la régulation des comportements déviants en Éducation Physique et Sportive : une comparaison entre enseignants titulaires et stagiaires. Mov Sport Sci/Sci Mot, Vol, 180002s 